\title{
Dos nuevas especies del género Squatina (Chondrichthyes: Squatinidae) del Golfo de México
}

\author{
José Luis Castro-Aguirre ${ }^{1}$, Héctor Espinosa Pérez ${ }^{2}$ \& Leticia Huidobro Campos ${ }^{2}$ \\ 1 Centro Interdisciplinario de Ciencias Marinas, IPN, Colección Ictiológica. Apdo. 592, La Paz, Baja California Sur, \\ México 23001; jlcastroaguirre@hotmail.com \\ 2 Instituto de Biología, UNAM, Colección Nacional de Peces. Apdo. 70-153, México 04510, D.F., México; \\ hector@servidor.unam.mx; huidobro@ibiologia.unam.mx
}

Recibido 30-VII-2003. Corregido 02-VI-2005. Aceptado 11-V-2006.

\begin{abstract}
Two new species of the genus Squatina (Chondrichthyes: Squatinidae) from the Gulf of Mexico. Two undescribed species of the genus Squatina, caught by bottom-trawl during the OGMEX VIII, IX and PROBEMEX II oceanographic cruises were compared with S. dumeril Lesueur, 1818, the only well known species from the northern Gulf of Mexico. The collections were made off Tamaulipas, Veracruz and Tabasco. The descriptions of the new species refer to morphology, coloration pattern and dorsal fin shape and size. An English description of each species is included. Some specimens erroneously assigned to $S$. dumeril are deposited in Mexican collections. With these two new species, besides S. californica Ayres, 1859 and S. dumeril , the number of documented species of this genus in Mexico ascends to four, and a total of five are known from the western Atlantic. A key is provided for their identification. Rev. Biol. Trop. 54 (3): 1031-1040. Epub 2006 Sept. 29.
\end{abstract}

Key words: Chondrichthyes, Squatina, Gulf of Mexico, key.

El género Squatina contiene alrededor de 11 a 14 especies nominales cuya distribución se localiza en zonas templadas y subtropicales del océano mundial. Son tiburones de cuerpo deprimido, muy parecidos a los batoideos y de hábitos bénticos cuya alimentación gravita en lo esencial sobre rayas, peces e invertebrados. Su semejanza con los batoideos parece ser resultado de un proceso de convergencia evolutiva más que de una relación filogenética cercana. El relativamente poco conocimiento del grupo se refleja en el número tan variable de especies descritas.

Compagno (1984) ofreció una clave provisional para su identificación elaborada con base en la correspondiente de Bigelow y Schroeder (1948) y aunque ambas intentan ser de utilidad en la discriminación de todas las conocidas, no logran conseguir ese objetivo, debido a la gran homogeneidad morfológica que manifiestan. Previo a esto, Garman (1913) proporcionó una clave de alcance semejante; Marini (1936), Fowler (1941), Bass et al. (1975), Castro (1983) y Roux (1984) hicieron lo propio aunque bastante restringidas en su cobertura geográfica. Por su parte, Vooren y Da Silva (1991) incluyeron diagnosis de las tres formas que se localizan frente a los litorales del sur de Brasil y Argentina. En las costas de México se habían reconocido hasta ahora dos: Squatina californica Ayres, 1859 que se localiza desde Alaska hasta Cabo San Lucas y Golfo de California (con una población discontinua en Ecuador, Perú y Chile) y $S$. dumeril Lesueur, 1818 que se distribuye desde Nueva Inglaterra, EEUU hasta la parte norte del Golfo de México y con varios registros en Jamaica, Nicaragua y Venezuela (McEachran y Fechhelm 1998). Su presencia en el litoral mexicano se encuentra mal definida y es probable que no forme parte de su condrictiofauna. En fecha reciente, Espinosa Pérez et al. (2004) proporcionaron una sinopsis para 
separar, tanto a las conocidas como a las no descritas, designándoseles como Squatina sp. A y Squatina sp. B. Aquí se incluye una clave para su identificación, con algunas modificaciones, en el contexto de esta contribución. El estudio de varios individuos pertenecientes al género Squatina, capturados con red de arrastre durante las campañas oceanográficas OGMEX (Oceanografía del Golfo de México) VIII, IX y PROBEMEX (Prospección Bentónica de Golfo de México) II, a bordo del Buque Oceanográfico de la UNAM “Justo Sierra", frente a las costas de Tamaulipas, Veracruz y Tabasco (ver datos precisos en las páginas siguientes) permitió detectar la existencia de dos especies no descritas, que a continuación se tratan (Cuadro 1). Para su descripción se analizaron morfológica y merísticamente ejemplares catalogados como $S$. dumeril y depositados en la Colección de Ictiología de la Facultad de Ciencias Biológicas, Universidad Autónoma de Nuevo León, en Monterrey, Nuevo León, México; así como individuos de S. californica de la Colección Ictiológica del Centro Interdisciplinario de Ciencias Marinas, I.P.N. en La Paz, Baja California Sur, México(CICICIMAR, IPN), de la Colección Nacional de Peces en el Instituto de Biología (IBUNAMP), en México, D.F. y de la Colección de Peces de la Texas A\&M University en Austin, Texas, EEUU.

\section{Squatina mexicana sp. nov.}

(Fig. 1)

Material examinado: Holotipo, macho de $880 \mathrm{~mm}$ LP, IBUNAM-P 8306, plataforma continental de Tamaulipas, 22 ${ }^{\circ} 58^{\prime} 05^{\prime \prime} \mathrm{N}$ y 97²8’07” W, 11 de septiembre de 1989. Prof. 71 m. Fauna acompañante: Ancylopsetta dilecta, Bothus robinsi, Brotula barbata, Caulolatilus intermedius, Equetus iwamotoi, Haemulon aurolineatum, Halieuthichtys aculeatus, Kathetostoma albigutta, Lepophidium brevibarbe, Ogcocephalus radiatus, Porichthys plectrodon, Saurida caribbaea, Scorpaena dispar, Serranus atrobranchus, Sphoeroides maculatus, Sphoeroides nephelus, Sygnathus

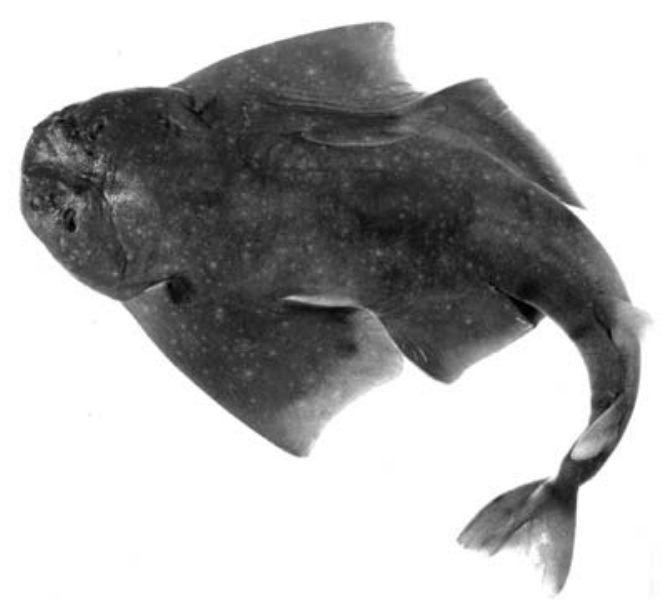

Fig. 1. Squatina mexicana sp. nov.

caribbaeus, Trichopsetta ventralis, Upeneus parvus y Urophycis floridana.

Paratipos: Hembra de $418 \mathrm{~mm}$ LP, macho de 329 mm LP, macho de 334 mm LP, macho de 232 mm LP. IBUNAM-P 11136. Plataforma continental frente a Tabasco en el Golfo de México, 1841'80” N y 9250’60” W, 10 de marzo de 1999. Prof. $101 \mathrm{~m}$. Macho de 502 mm LP. IBUNAM-P 12723. Plataforma continental frente a Yucatán, México, 2309'35”N y 89³2'49”'W, 25 de abril de 1993. Prof. 177-180 m. Macho de 490 mm LP. IBUNAM-P 12724. Plataforma continental frente a Tabasco en el Golfo de México, 1845’28”' N y 93³2’35” W, 13 de marzo de 1999. Prof. 96 m.

Diagnosis: Sin espinas ni aguijones sobre la línea media del dorso. Dentículos dérmicos provistos de tres quillas que se extienden posteriormente (Fig. 2); su base angosta, aproximadamente cuatro veces en su longitud. Aletas dorsales semejantes en tamaño, forma y área, su base alrededor de 1.75 veces en su altura (Fig. 3). Lóbulos nasales simples, el anterior más largo y delgado que el posterior; el central cuadrangular y por poco desprovisto de lóbulos (Fig. 4). Coloración general gris, sin ocelos, con dos manchas negras conspicuas sobre el borde 
CUADRO 1

Medidas morfométricas ( $\mathrm{mm}$ ) de ejemplares de Squatina

TABLE 1

Morphometric measures $(\mathrm{mm})$ for specimens of Squatina

S. mexicana sp.nov.

Sexo

Medidas

Longitud total

Longitud patrón

Longitud de la cabeza

Diámetro horizontal del ojo

Distancia del hocico al ojo

Distancia del ojo al espiráculo

Distancia entre los ojos

Distancia entre los espiráculos

Amplitud de la cabeza frente

a los espiráculos

Amplitud de la cabeza frente a los ojos

Amplitud posterior de la cabeza

Amplitud de la boca

Distancia entre las bases

posteriores de las pectorales

Amplitud de las pectorales

Distancia entre las bases

posteriores de las pélvicas

Distancia del hocico a la axila posterior de las pectorales

Distancia del hocico a la base

de las pectorales

Margen posterior de la pectoral

Base de la pectoral

Distancia del hocico a la 1a. dorsal

Distancia entre la 1a. y 2a. dorsal

Distancia entre el fin de la 2a. dorsal y la caudal

Base de la 1a. dorsal $\begin{array}{llllllllllll}\text { M } & \text { M } & \text { M } & \text { H } & \text { H } & \text { M } & \text { M } & \text { M } & \text { M } & \text { M } & \text { H } & \text { M }\end{array}$

$\begin{array}{llllllllllll}930 & 568 & 570 & 423 & 361 & 380 & 393 & 380 & 585 & 347 & 321 & 355 \\ 880 & 502 & 490 & 372 & 418 & 329 & 334 & 232 & 490 & 295 & 270 & 302 \\ 196 & 113 & 122 & 92 & 81 & 77 & 77 & 77 & 121 & 71 & 71 & 72 \\ 16.5 & 10 & 9 & 7 & 8 & 8 & 8 & 8 & 12 & 8 & 6 & 6 \\ 56 & 32 & 33 & 25 & 15 & 14 & 14 & 10 & 19 & 13 & 11 & 7 \\ 27 & 15 & 15 & 11 & 11 & 10 & 11 & 11 & 16 & 12 & 8 & 6 \\ 76.5 & 50 & 49 & 34 & 35 & 33 & 33 & 33 & 49 & 31 & 27 & 32 \\ 68 & 48 & 45 & 35 & 34 & 30 & 31 & 30 & 45 & 28 & 26 & 29\end{array}$

$\begin{array}{llllllllllll}208 & 119 & 120 & 84 & 82 & 71 & 77 & 74 & 114 & 74 & 65 & 70\end{array}$

$\begin{array}{llllllllllll}242 & 137 & 136 & 96 & 57 & 46 & 50 & 48 & 140 & 47 & 46 & 48\end{array}$

$\begin{array}{llllllllllll}84 & 84 & 84 & 66 & 68 & 59 & 59 & 57 & 84 & 56 & 49 & 53\end{array}$

$\begin{array}{llllllllllll}143 & 87 & 84 & 58 & 55 & 49 & 52 & 50 & 73 & 50 & 42 & 46\end{array}$

$\begin{array}{llllllllllll}129 & 100 & 99 & 70 & 68 & 69 & 65 & 59 & 107 & 64 & 49 & 55\end{array}$

$\begin{array}{llllllllllll}595 & 320 & 300 & 215 & 185 & 192 & 183 & 192 & 334 & 180 & 135 & 167\end{array}$

$\begin{array}{llllllllllll}121 & 72 & 75 & 47 & 46 & 42 & 47 & 49 & 77 & 41 & 35 & 57\end{array}$

$\begin{array}{llllllllllll}266 & 174 & 172 & 133 & 127 & 114 & 121 & 113 & 170 & 159 & 97 & 101\end{array}$

$\begin{array}{lllllll}189 & 114 & 113 & 85 & 125 & 60 & 68\end{array}$

$\begin{array}{llllllllllll}274 & 156 & 164 & 116 & 115 & 122 & 93 & 122 & 168 & 100 & 106 & 112\end{array}$

$\begin{array}{llllllllllll}117 & 71 & 74 & 46 & 49 & 43 & 31 & 31 & 56 & 32 & 39 & 36\end{array}$

$\begin{array}{llllllllllll}620 & 353 & 369 & 273 & 264 & 239 & 246 & 254 & 371 & 214 & 203 & 218\end{array}$

$\begin{array}{llllllllllll}65 & 37 & 40 & 24 & 28 & 24 & 24 & 235 & 36 & 24 & 21 & 22\end{array}$

$\begin{array}{llllllllllll}72 & 41 & 42 & 32 & 28 & 28 & 34 & 30 & 47 & 24 & 83 & 30\end{array}$

$\begin{array}{llllllllllll}44 & 24 & 26 & 14 & 16 & 16 & 15 & 15 & 25 & 15 & 12 & 13\end{array}$ 
CUADRO 1 (...continued)

Medidas morfométricas ( $\mathrm{mm}$ ) de ejemplares de Squatina

TABLE 1 (...continuación)

Morphometric measures $(\mathrm{mm})$ for specimens of Squatina

\begin{tabular}{|c|c|c|c|c|c|c|c|c|c|c|c|c|}
\hline \multirow[b]{2}{*}{ Altura de la 1a. dorsal } & \multicolumn{8}{|c|}{ S. mexicana sp.nov. } & \multicolumn{2}{|c|}{$\begin{array}{l}\text { S.heteroptero } \\
\text { sp. nov. }\end{array}$} & \multicolumn{2}{|c|}{ S. dumeril } \\
\hline & 81 & 39 & 39 & 27 & 17 & 20 & 19 & 21 & 41 & 17 & 19 & 23 \\
\hline Base de la 2a. dorsal & 39 & 24 & 23 & 14 & 14 & 14 & 14 & 14 & 24 & 14 & 11 & 12 \\
\hline Altura de la 2a. dorsal & 71 & 39 & 40 & 25 & 16 & 16 & 18 & 18 & 42 & 17 & 18 & 21 \\
\hline $\begin{array}{l}\text { Distancia entre el ápice anterior al ápice } \\
\text { interno de la pectoral }\end{array}$ & 200 & 169 & 110 & 75 & 74 & 68 & 66 & 69 & 111 & 60 & 62 & 64 \\
\hline
\end{tabular}

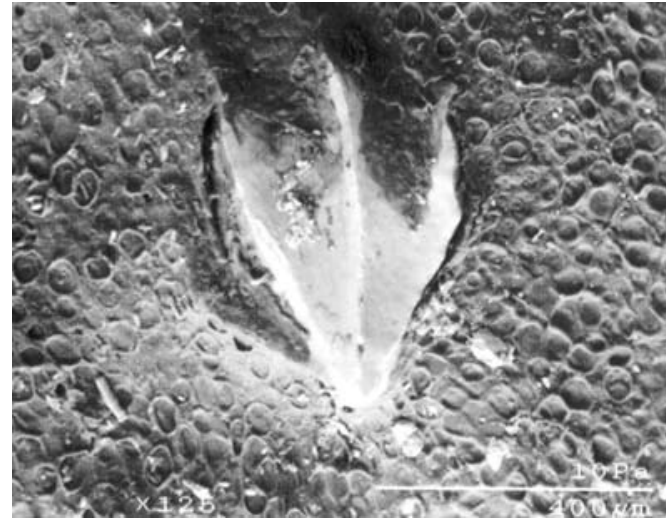

Fig. 2. Dentículos dérmicos de Squatina mexicana sp. nov.

Fig. 2. Dermal denticles of Squatina mexicana sp. nov.

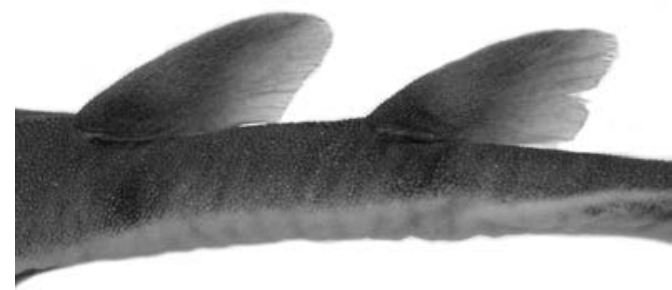

Fig. 3. Aletas dorsales de Squatina mexicana sp. nov.

Fig. 3. Dorsal fins of Squatina mexicana sp. nov.

anterior de las aletas pectorales; en general el dorso con pequeñas manchas oscuras diseminadas en forma irregular. Dentición: ambos lados de la mandíbula superior con diez dientes triangulares, erectos y bordes no aserrados,

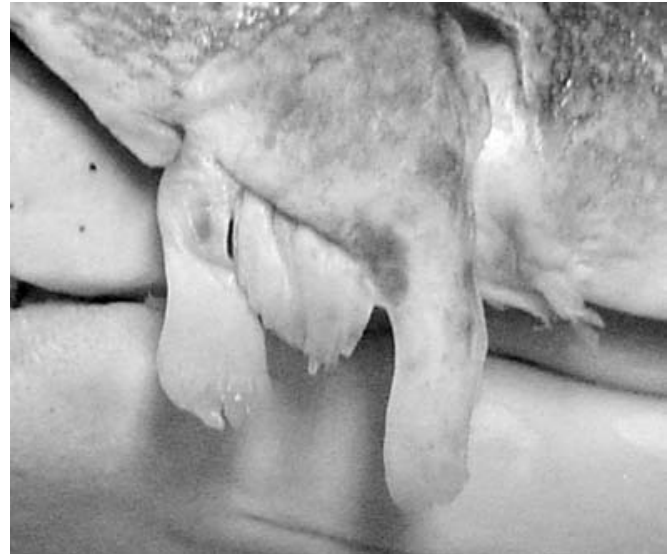

Fig. 4. Lóbulos nasales de Squatina mexicana sp. nov.

Fig. 4. Nasal lobes of Squatina mexicana sp. nov.

dispuestos en dos series funcionales. Mandíbula inferior con nueve a diez dientes triangulares y bordes lisos. Los cinco primeros son erectos, los restantes son ligeramente oblicuos. También se disponen en dos series funcionales.

English diagnosis: Without thorns or enlarged denticles on the mean dorsal line. Dermal skin denticles with three keels extended posteriorly (Fig. 2), its base approximately four times in its length. Dorsal fins similar in size, shape and area, their base about 1.75 times in their height (Fig. 3). Nasal lobes simple, the anterior thin and longer; the medium lobe is quadrangular and almost lacking lobes (Fig. 
4). Color is gray, without ocelli, but there are two very evident black spots on the anterior edge of pectoral fins; the rest of body black with small scattered dark spots in irregular disposition. Both sides of upper jaw with 10 triangular, erect, and non edge-serrated teeth; the teeth are arranged in two functional series; lower jaw with nine or ten smooth triangular teeth. The five frontal teeth are straight, the others are slightly oblique and arranged in two functional series.

Descripción: Cabeza y cuerpo deprimidos en toda su longitud; región cefálica amplia, su amplitud máxima, mayor que la longitud cefálica, su mínima amplitud muy aproximada a la longitud cefálica. Cuerpo ancho, su anchura máxima cercana a la longitud cefálica; pedúnculo caudal adelgazado progresivamente hacia atrás, su ancho máximo se localiza entre los márgenes internos de las aletas pélvicas y es mayor que la distancia interespiracular; su ancho mínimo se localiza en el origen de la aleta caudal. Las quillas laterales caudales están bien desarrolladas, su ancho máximo es poco más o menos el doble de la distancia interocular.

Región cefálica ampliamente redondeada, en forma de parábola en vista dorsal; boca terminal y amplia, su extensión máxima mayor que la distancia interespiracular. Borde dérmico superior de la mandíbula superior, adherido a la región cefálica solamente en la zona terminal de ambas mandíbulas. Su porción central presenta una muesca en forma de arco muy evidente y permite observar el frente de la mandíbula superior. Orificios nasales situados inmediatamente por arriba de las mandíbulas, provistos de un reborde dérmico triangular dirigido hacia abajo; el lóbulo nasal superior consiste de dos prolongaciones simples en forma de espátula, pero con sus ápices levemente festoneados y separados por una estructura cuadrangular simple con su borde ligeramente ondulado. Ojos en forma oval, su diámetro horizontal con escasa diferencia a la longitud interna de los espiráculos. Cada lado de la mandíbula superior provisto de diez dientes triangulares, erectos y de bordes lisos $\mathrm{y}$ en dos series de dientes funcionales. Cada lado de la mandíbula inferior con nueve a diez dientes triangulares y de bordes lisos, en dos series funcionales, los cinco primeros con sus cúspides erectas y los restantes se disponen de forma ligeramente oblícua. Sin dientes en la sínfisis de ambas mandíbulas.

Aguijones cefálicos dispuestos de la manera siguiente: tres sobre la mandíbula superior, cerca de los extremos del arco mandibular; uno a cada lado de la cabeza anteriores al ojo, justo a la mitad de la distancia que existe entre el poro nasal y el margen anterior del ojo; dos sobre el margen supraposterior de cada ojo; uno a cada lado del área interespiracular, muy notables y desarrollados.

Poros endolinfáticos situados aproximadamente al mismo nivel de los espiráculos y a la mitad de la distancia interespiracular. Antes de cada poro existe un canal bastante visible y ambos forman una estructura en forma de "V".

Aletas pectorales grandes, su borde anterior convexo, el externo recto o ligeramente convexo, el posterior e interno cóncavo. Su base con corta diferencia a la distancia interocular. Las aletas pectorales cubren ligeramente al borde anterior de las pélvicas, el cual es levemente convexo, el posterior recto o ligeramente convexo y el interno recto. Aletas dorsales semejantes en forma y tamaño, triangulares, su margen anterior es convexo, el posterior e interno recto. Aleta caudal ligeramente heterocerca, su lóbulo inferior es ligeramente más grande que el superior. Sin aleta anal.

Lado dorsal de la región cefálica y del cuerpo, así como las aletas dorsales, pectorales, pélvicas y caudal completamente cubiertas por dentículos dérmicos. Región ventral de la cabeza desprovista de dentículos dérmicos, así como el tórax y vientre, con excepción de algunas áreas como las aletas pectorales y pélvicas, donde se presentan en zonas muy dispersas. En el lado ventral del pedúnculo caudal, los dentículos cubren un área que incluye hasta la parte media de las aletas pélvicas, aunque existe un área aproximadamente en forma de $\mathrm{V}$ (cuyo ápice se localiza antes de la punta posterior de las aletas pélvicas), desprovista de estas estructuras. 
Coloración: Región dorsal de color pardo uniforme, con cuatro barras equidistantes de color pardo oscuro o negro en la región caudal: la primera y la más anterior, se localiza a nivel de la mitad posterior de las aletas pélvicas, la segunda se localiza en la parte media de la primera aleta dorsal, la tercera en la segunda dorsal, la cuarta (muy débil) en el pedúnculo caudal. Aletas pectorales con una mancha negra redondeada (muy débil) sobre la axila anterior. Otra mancha negra redondeada, también muy débil, que se sitúa en la parte posterior de cada aleta pectoral.

Derivación del nombre específico: Alude a México, en cuyo golfo fueron capturados los ejemplares base de la presente descripción.

Localidades conocidas: Plataforma continental frente a Tabasco en el Golfo de México, 1841'80” N y 9250'60” W, 10 de marzo de 1999. Prof. $101 \mathrm{~m}$. IBUNAM-P 11136. Plataforma continental frente a Tamaulipas en el Golfo de México, 2309'35”' N y 89³2'47”' W, 25 de abril de 1993. IBUNAM-P 12723. Plataforma continental frente a Tabasco en el Golfo de México, 1845'28”' N y 9332'35”' W, 13 de marzo de 1999. Prof. 96 m. IBUNAM-P 12724.

\section{Squatina heteroptera sp. nov.}

(Fig. 5)

Material examinado: Holotipo, macho de 490 mm LP, IBUNAM-P 8307. Plataforma continental de Tamaulipas, en el Golfo de México, 2244'1” N y 97²4'7” W, 13 de septiembre

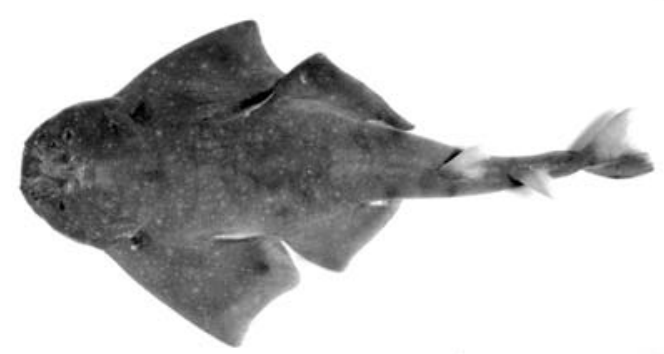

Fig. 5. Squatina heteroptera sp. nov. de 1989. Prof. 157-164 m. Fauna acompañante: Calamopteryx robinsorum, Dyssomma aphododera, Hypsicometes gobioides, Lepophidium brevibarbe, Monolene sessilicauda, Neoepinnula orientalis, O. radiatus, Polymixia lowei, Pontinus longispinis, Pristipomoides aquilonaris, Symphurus plagiusa, Trachurus lathami, T. ventralis y Urophycis cirratus.

Paratipos: Macho de $295 \mathrm{~mm}$ LP. IBUNAM-P 12722. Plataforma continental frente a Tabasco en el Golfo de México, 1841'80” N y 9250'60" W, 10 de marzo de 1999. Prof. $101 \mathrm{~m}$.

Diagnosis: Sin espinas ni aguijones sobre la línea media del dorso. Dentículos dérmicos provistos de cuatro quillas que se extienden posteriormente (Fig. 6); su base ancha, aproximadamente dos veces su longitud. Aletas dorsales diferentes en tamaño, forma y área, su base alrededor de la mitad de su altura (Fig. 7). Lóbulos nasales simples e iguales en su longitud; el anterior y posterior en forma de espátula; el central de forma cuadrangular irregular y con varios lóbulos (Fig. 8). Coloración general pardo oscuro en el dorso, sin ocelos, pero con dos manchas negras conspicuas sobre el borde anterior de

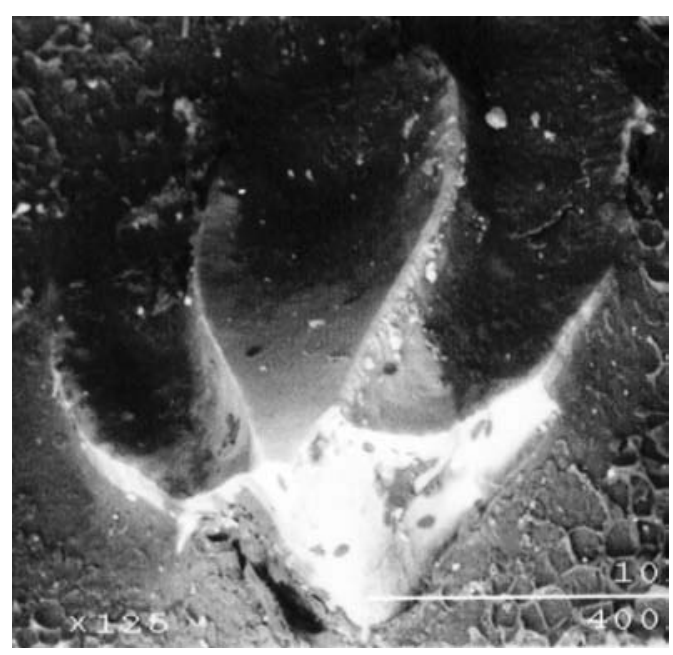

Fig. 6. Dentículos dérmicos de Squatina heteroptera sp. nov.

Fig. 6. Dermal denticles of Squatina heteroptera sp. nov. 


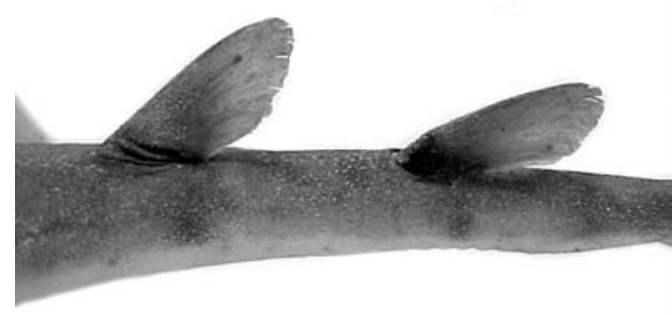

Fig. 7. Aletas dorsales de Squatina heteroptera sp. nov.

Fig. 7. Dorsal fins of Squatina heteroptera sp. nov.

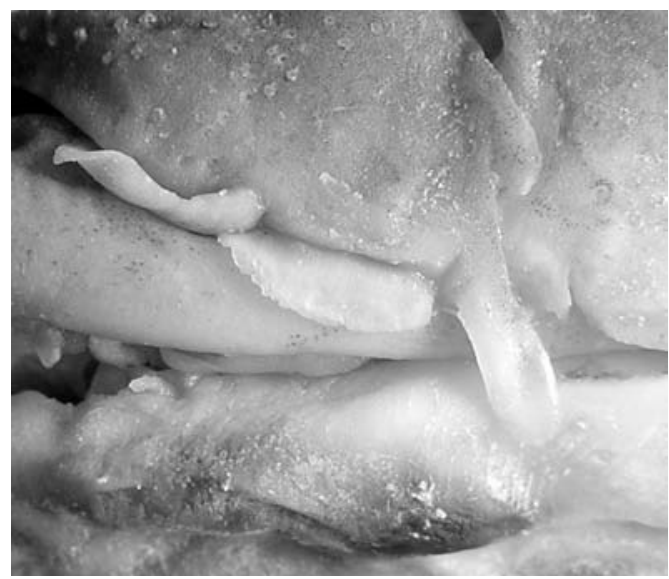

Fig. 8 Lóbulos nasales de Squatina heteroptera sp. nov.

Fig. 8. Nasal lobes of Squatina heteroptera sp. nov

las aletas pectorales; dorso con pequeñas manchas blanquecinas diseminadas irregularmente. Dentición: ocho dientes triangulares y erectos a cada lado de ambas mandíbulas, la superior con dos series funcionales, la inferior con una. Todas ellas sin aserraciones a los lados.

English diagnosis: Without thorns or enlarged denticles on the mean dorsal line. Dermal denticles with four keels extend posteriorly (Fig. 6), its widest base, about twice its length. Dorsal fins very different in size, shape and area, their base approximately half of their height (Fig. 7). Nasal lobes are simple and similar in its length; the first and the last one, are spatulated-like shape; the central part is irregularly quadrangular and its edge with several lobes (Fig. 8). General color is dark brown in dorsal surface without ocelli, but with two evident round black spots on the upper edge of pectoral fins and with irregular and scattered whitish spots. Eight triangular, nonserrated, and straight teeth in each side of jaws, the upper with two functional series, the lower with only one.

Descripción: Cabeza y cuerpo deprimidos en toda su longitud; región cefálica amplia, su máxima extensión mayor que la longitud cefálica, su mínima amplitud aproximada a la longitud cefálica. Cuerpo ancho, su máxima extensión igual a la longitud cefálica; pedúnculo caudal adelgazado progresivamemente hacia atrás, su ancho máximo se localiza entre los márgenes internos de las aletas pélvicas y es cerca de la distancia interespiracular; su ancho mínimo se localiza en el origen de la aleta caudal. Las quillas laterales caudales están bien desarrolladas, su ancho máximo es aproximado al doble de la distancia interocular.

Región cefálica ampliamente redondeada, en forma de parábola en vista dorsal; boca terminal y amplia, su máxima extensión igual o con escasa diferencia a la distancia interespiracular. Borde dérmico superior de la mandíbula superior adherido a la región cefálica, solamente en la zona terminal de ambas mandíbulas. La porción central presenta una muesca en forma de arco muy evidente y permite observar el frente de la mandíbula superior. Orificios nasales muy notables, situados inmediatamente hacia arriba de las mandíbulas, provistos de un reborde dérmico triangular dirigido hacia abajo; el lóbulo nasal superior consiste de dos prolongaciones simples en forma de espátula, separadas por una estructura cuadrangular simple y por poco desprovista de rebordes. Ojos en forma oval, su diámetro horizontal con escasa diferencia a la longitud interna máxima de los espiráculos. Cada lado de la mandíbula superior provisto de ocho dientes triangulares y erectos a cada lado dispuestos en dos series funcionales. Cada lado de la mandíbula inferior con ocho dientes en una sola serie, erectos los tres primeros, los cinco restantes con la cúspide ligeramente dirigida hacia los lados. Sínfisis 
de ambas mandíbulas desprovistas de diente central. Aguijones cefálicos dispuestos de la siguiente manera: dos sobre la mandíbula superior, cerca de los extremos del arco mandibular; uno a cada lado de la región cefálica, justo a la mitad de la distancia que existe entre el poro nasal y el margen anterior del ojo; uno a cada lado de la cabeza, anteriores al borde anterior del ojo; un grupo circular de cuatro aguijones muy notables y de dos a tres de menor tamaño, situado atrás y arriba de cada ojo, el área conformada por este grupo, se encuentra desprovista de dentículos dérmicos; uno muy notable y desarrollado a cada lado de los espiráculos. Poros endolinfáticos situados hacia atrás de los espiráculos a mitad de la distancia interespiracular. Canales de los poros endolifáticos en forma de "U”. Espiráculos grandes, en forma de riñon, situados atrás de los ojos, su máxima amplitud aproximada al diámetro horizontal del ojo. Cinco aberturas branquiales laterales, aunque se continúan hacia la parte ventral de la cabeza, situadas sólo en la región cefálica.

Aletas pectorales grandes, su borde anterior levemente convexo o recto, el posterior cóncavo y el interno convexo. Su base igual o mayor que la distancia interocular. Las aletas pectorales no cubren a las pélvicas. Éstas últimas tienen su borde anterior convexo, el posterior e interno recto o levemente convexo. Aletas dorsales no semejantes en forma: la primera es triangular, su margen anterior recto o levemente convexo y redondeado en su ápice al igual que el superior, el posterior recto. La segunda dorsal es oval, su margen anterior convexo, su ápice es redondeado, el posterior levemente cóncavo o recto y el interno convexo. Aleta caudal ligeramente heterocerca, su lóbulo inferior algo mayor que el superior. Sin aleta anal.

Lado dorsal del cuerpo, región cefálica, aletas pectorales, pélvicas y dorsales completamente cubiertos por dentículos dérmicos. Región ventral de la cabeza desprovista de dentículos dérmicos, así como el tórax y vientre, con excepción de algunas áreas muy puntuales de las aletas pectorales y pélvicas. La parte ventral del pedúnculo caudal presenta dentículos dérmicos en un área que alcanza justo antes del ápice de las aletas pélvicas.

Coloración: Región dorsal de color pardo o gris oscuro, aletas pectorales con dos manchas negras, muy visibles cerca de las axilas; pedúnculo caudal con tres manchas oscuras a cada lado: un par sobre las aletas pélvicas, otro justo a nivel de la primera dorsal y el último abajo de la segunda dorsal. Región ventral de color amarillo o blanquecino y sin manchas.

Derivación del nombre específico: Alude a la obvia diferencia que presentan las dos aletas dorsales, en cuanto a su forma, tamaño y área.

Localidades conocidas: Plataforma continental del Golfo de México, 1841'80” N y 9250’60” W, 10 de marzo de 1999. Prof. 101 m. IBUNAM-P 12722.

\section{DISCUSIÓN}

Las dos especies aquí descritas pertenecen al grupo taxonómico que se caracteriza por tener las barbillas nasales anteriores simples, no bifurcadas o festoneadas en extremo (Bigelow y Schroeder 1948, Compagno 1984), además de no poseer dentículos dérmicos en forma de aguijones piramidales a lo largo de la línea media del dorso ni entre las aletas dorsales, a diferencia de las otras dos especies conocidas o citadas de los litorales de México (S. californica Ayres y S. dumeril Lesueur) (Fig. 9, 10, Cuadro 1). La ausencia de aguijones en la línea media del dorso también es compartida con Squatina

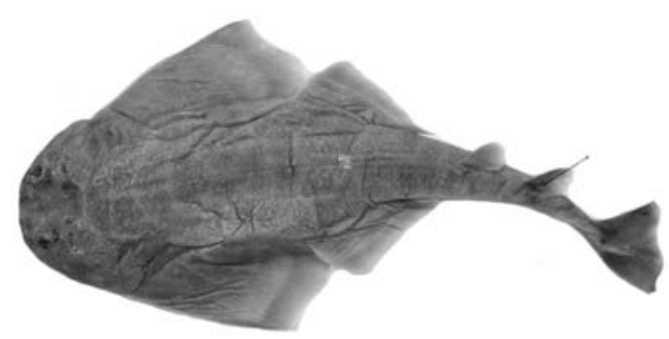

Fig. 9. Squatina californica Ayres. 


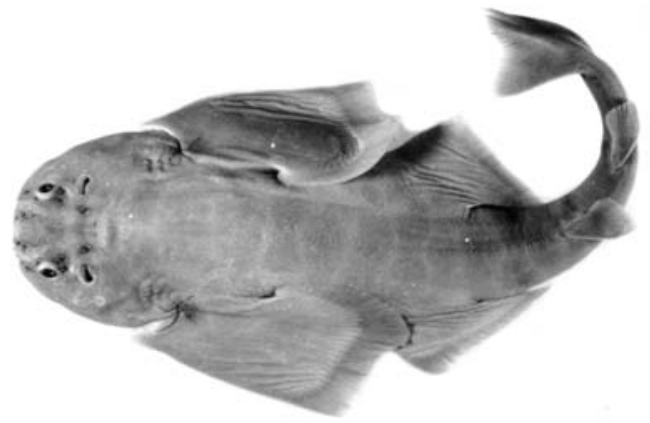

Fig. 10. Squatina dumeril Lesueur.

argentina (Marini, 1930) y S. occulta Vooren y Da Silva, 1991, que se localizan frente a las costas de Brasil y Argentina. Se elaboró la siguiente clave para identificar a las especies conocidas del género Squatina en el Atlántico occidental, derivada de las contribuciones de Vooren y Da Silva (1991) y Espinosa Pérez et al. (2004):

\section{AGRADECIMIENTOS}

Agradecemos a Luis Soto-González y a Carlos Illescas por su invitación a participar en las campañas oceanográficas OGMEX y PROBEMEX, del Instituto de Ciencias del Mar y Limnología, UNAM. De igual forma a John D. McEachran de la Universidad de Texas A \& M, José de la Cruz-Agüero y Adrián González-Acosta del Centro Interdisciplinario de Ciencias Marinas del IPN por información, ejemplares y fotografias facilitados de Squatina spp., a Angélica Daza por su ayuda con el cuadro. También a M. Lourdes Lozano-Vilano de la Universidad Autónoma de Nuevo León, quien permitió analizar ejemplares de $S$. dumeril de la colección a su cargo, a Carmen Loyola por las fotografías de varios individuos y a un revisor anónimo por sus comentarios, que ayudaron a mejorar el trabajo.

1. Parte media del dorso con una serie de dentículos dérmicos modificados como aguijones recurvados hacia atrás. . . 2

-- $\quad$ Parte media del dorso sin dentículos dérmicos como los descritos anteriormente $\ldots \ldots \ldots \ldots \ldots \ldots$

2. Borde posterior de las aletas pectorales cóncavo, alcanzan el borde anterior de las pélvicas pero no las cubren; aletas dorsales de forma más o menos triangular, la base de la primera menor que la base de la segunda; fórmula dentaria: 10-10/10-10 a 11-11/11-11; superficie dorsal de color pardo claro uniforme con manchas negras circulares dispuestas de manera simétrica. Especie conocida desde Río de Janeiro, Brasil hasta el sur de la Provincia de Buenos Aires, Argentina. . . . . . . . . . . . . . . . . . . . . . . . . Squatina guggenheim Marini, 1936

-- Borde posterior de las aletas pectorales no demasiado cóncavo, alcanzan a rebasar al borde anterior de las pélvicas; aletas dorsales de forma oval, semejantes en tamaño y área, sus bases de igual longitud; fórmula dentaria: 10-10/9-9; superficie dorsal de color pardo oscuro con numerosas manchas negras dispuestas de manera irregular. Especie conocida desde el sur de Nueva Inglaterra, E.U.A. hasta Antillas y Venezuela, inclusive el Golfo de México. . . . . . . . . .

Squatina dumeril Lesueur, 1818

3. Aletas pectorales relativamente cortas, su borde interno no alcanza el margen anterior de las pélvicas; fórmula dentaria: de 9-9/10-10 a 10-10/11-11. Especie conocida desde la costa centro sur de Brasil hasta el sur de la provincia de Buenos Aires, Argentina . . . . . . . . . . . . . . . . . . . . . . . . Squatina occulta Vooren y Da Silva, 1991

-- $\quad$ Aletas pectorales relativamente largas, su borde interno alcanza o rebasa el margen anterior de las pélvicas; fórmula

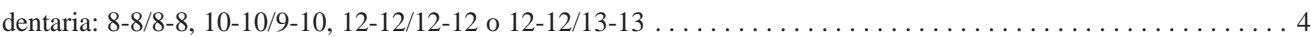

4. Aletas dorsales diferentes en forma, tamaño y área; fórmula dentaria: 8-8/8-8. Especie conocida en el Golfo de

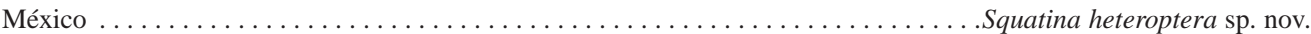

-- $\quad$ Aletas dorsales semejantes en forma tamaño y área. Una especie conocida del Golfo de México y otra desde el sur de Brasil hasta la porción centro norte de Argentina. 5

5. Fórmula dentaria: 10-10/9-10 o 10-10/10-10. Especie conocida del Golfo de México ... Squatina mexicana sp. nov.

-- Fórmula dentaria: 12-12/12-12 o 12-12/13-13. Especie conocida desde el sur de Brasil hasta la porción centro norte de

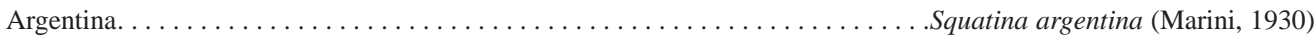




\section{RESUMEN}

El estudio de varios individuos pertenecientes al género Squatina, capturados con red de arrastre durante las campañas oceanográficas OGMEX VIII, IX y PROBEMEX II, a bordo del Buque Oceanográfico de la UNAM “Justo Sierra” frente a las costas de Tamaulipas, Veracruz y Tabasco, permitió detectar la existencia de dos especies no descritas, que fueron comparadas con S. Dumeril Lesueur, 1818, única especie en el norte del Golfo de México. Las descripciones de las nuevas especies hacen referencia a su morfología, patrón de coloración, forma y tamaño de las aletas dorsales principalmente. Algunos ejemplares, erróneamente asignados como S. dumeril, se encuentran depositados en colecciones de México. Con estas dos nuevas especies, además de $S$. dumeril y S. Californica Ayres, 1859, son cuatro las que se detectan dentro del territorio mexicano y cinco las que existen en el Atlántico Occidental. Se incluye una clave para su identificación.

Palabras clave: Chondrichthyes, Squatina, Golfo de México, clave.

\section{REFERENCIAS}

Bass, A.J., J.D. D’Aubrey \& N. Kistnasamy. 1975. Sharks of the east coast of southern Africa. V. The families Hexanchidae, Chlamydoselachidae, Heterodontidae, Pristiophoridae and Squatinidae. South Africa Oceanogr. Res., Inst. Invest. Rept. 43: 1-50.

Bigelow, H.B. \& W.C. Schroeder. 1948. Sharks. In Fishes of the Western North Atlantic. Mem. Sears Found. Mar. Res. 1: 59-576.

Castro, J.I. 1983. The sharks of North American waters. Texas A \& M University, Austin, Texas, EEUU. $x+180$ p.
Compagno, L.V.J. 1984. FAO species catalogue. Vol. 4. Sharks of the world. An annotated and illustrated catalogue of sharks species known to date. Pt. 1. Hexanchiformes to Lamniformes. FAO Fish. Synopsis, (125) vol. 4, pt. 1: viii + 249 p.

Espinosa Pérez, H.S., J.L. Castro-Aguirre \& L. Huidobro Campos. 2004. Listados Faunísticos de México. IX. Catálogo sistemático de tiburones (Elasmobranchii: Selachimorpha). México, D.F. Pub. Esp. Instituto de Biología, UNAM. 134 p.

Fowler, H.W. 1941. Contributions to the biology of the Philippine Archipelago and adjacent regions. In The fishes of the groups Elasmobranchii, Holocephali, Isospondyli and Ostariophysi obtained by the United States Bureau of Fisheries Steamer "Albatross" in 1907 to 1919, chiefly in the Philippine Islands and adjacent seas. Bull. U.S. Nat. Mus. 100: $\mathrm{x}+879$ p.

Garman, S. 1913. The Plagiostomia (sharks, skates and rays). Mem. Mus. Comp. Zool. 36: xiii + 528 p.

Marini, T.L. 1936. Revisión de las especies de la familia Squatinidae en las aguas argentinas (S. guggenheim n. sp.). Physis 12: 19-30.

McEachran, J.D. \& J.D. Fechhelm. 1998. Fishes of the Gulf of México. Vol. 1. Myxiniformes to Gasterosteiformes. Austin: University of Texas, EEUU. viii + 1112 p.

Roux, C. 1984. Squatinidae, p. 148-152. In P.J.P. Whitehead, M.L. Bauchot, J.C. Hureau, J. Nielsen \& E. Tortonese (eds.). Fishes of the North-East Atlantic and the Mediterranean. UNESCO, Paris, Francia. 510 p.

Vooren, C.M. \& K.G. Da Silva. 1991. On the taxonomy of the angel sharks from southern Brazil, with the description of Squatina occulta sp. n. Rev. Brasil. Biol. 51: 589-602. 